\title{
Acknowledgment to Reviewers of 2007
}

The editorial board expresses its sincere gratitude to the large group of reviewers of contributions to the journal Solar System Research. The successful work of the editorial board and the quality of the published articles directly depend on their dispassionate and selfless work. The following reviewers participated in the work of the journal in 2007:

DOI: $10.1134 / \mathrm{S} 0038094608010103$

V.K. Abalakin

P.B. Babadzhanov

A.V. Bagrov

Yu.V. Batrakov

O.I. Bel'kovich

A.I. Buikin

V.V. Busarev

V.M. Chepurova

N.N. Chugai

A.V. Devyatkin

V.N. Dudinov

A.I. Efimov

V.V. Emel'yanenko

N.V. Emel'yanov

E.G. Fateev

A.A. Fedorova

B.P. Filippov

S.A. Gasanov

M.V. Gerasimov

Kh.I. Ibadinov

V.N. Ishkov

A.V. Ivanov

M.A. Ivanov

G.S. Ivanov-Kholodnyi

V.V. Ivashkin

E.M. Kolesnikov

K.V. Kuimov

N.A. Lotova

D.F. Lupishko

H.V. Malova

V.L. Masaitis

L.I. Matveenko

Yu.D. Medvedev

A.V. Mel'nikov
L.I. Miroshnichenko

I.I. Mokhov

S.M. Molodensky

L.V. Moroz

Yu.A. Nagovitsyn

M.A. Nazarov

V.N. Obridko

E.V. Petrova

O.P. Popova

V.V. Prokof'eva

A.V. Rodin

Yu.Ya. Ruzhin

L.V. Rykhlova

V.A. Shefer

A.A. Shiryaev

V.A. Shor

Yu.A. Shukolyukov

V.V. Shuvalov

V.V. Sidorenko

N.S. Sidorenkov

B.V. Somov

Yu.I. Stozhkov

Yu.M. Torgashin

O.A. Troshichev

A.A. Ul'yanova

M.A. Vashkov'yak

V.V. Vidyakin

Yu.I. Voloshchuk

L.V. Zasova

S.G. Zhuravlev

On behalf of the editorial board,

M.Ya. Marov, Editor-in-Chief 